\title{
Valsartan reduces NOX4 expression and halts diabetic nephropathy in streptozotocin induced diabetic rat model
}

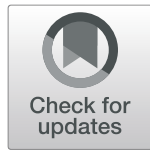

\author{
Shymaa A. Maher, Loaa A. Tag Eldeen, Dahlia I. Badran *iD and Taher I. Elserafy
}

\begin{abstract}
Background: Diabetic nephropathy is the leading cause of kidney failure worldwide. NADPH oxidase 4 (NOX4) is an obvious cause for reactive oxygen species production in the kidney mainly via renin-angiotensin system. High reactive oxygen species results in kidney damage through direct effect or through activation of other pathways such as the extracellular regulated kinase (ERK) signaling pathway.
\end{abstract}

Aim: The present study was constructed to investigate the effect of angiotensin II receptor blocker, valsartan, on NOX4, ERK1/2expression, and malondialdehyde level as well as their effects on the progression of diabetic nephropathy in type 1 diabetic rat model.

Methods: The experimental rats were divided into three groups: group I control healthy untreated rats, group II streptozotocin-induced diabetic rats, and group III valsartan treated streptozotocin-induced diabetic rats.

Results: Valsartan showed significantly decreased NOX4 and ERK1/2 mRNA, NOX4 and pERK1/2 protein concentration, and oxidative stress as evidenced by low malondialdehyde concentration in treated diabetic rats. Valsartan attenuates albuminuria, improve overall kidney function parameters, and pathological changes compared to diabetic non-treated rats.

Conclusion: Valsartan treatment impedes renal damage and improve kidney function in treated diabetic nephropathy rats. The beneficial effect of valsartan was mediated through decrease oxidative stress via downregulation of NOX4 and ERK1/2 and decreased reactive oxygen species production.

Keywords: NOX4, Diabetic nephropathy, Valsartan, ERK

\section{Background}

Diabetic nephropathy (DN), a major micro-vascular complication of diabetes, is the leading cause of end-stage renal disease worldwide (Collins et al., 2012; Gnudi et al., 2016). Chronic hyperglycemia, the most prominent feature of diabetes, direct the activation of intrarenal renin-angiotensin system (RAS), and increase production of reactive oxygen species (ROS), which leads to renal tissue injury and fibrosis (Urushihara and Kagami 2017; Murphy et al., 2015; Zhang et al., 1999; Singh et al., 1999) .

Angiotensin II (Ang II), a component of renin angiotensin system (RAS), mediate the development of diabetic nephropathy through various mechanisms (Ni et al., 2015;

\footnotetext{
* Correspondence: dahliabadran@hotmail.com

Department of Medical Biochemistry and Molecular Biology, Faculty of Medicine, Suez Canal University, Ismailia, Egypt
}

Onozato et al., 2002; Yadav et al., 2010). Ang II could increase ROS production through activating reduced nicotinamide adenine dinucleotide phosphate (NADPH) oxidase (NOXs) enzyme (Ushio-Fukai 2009), resulting in a state of oxidative stress and altered redox signaling, manifested by damage to macromolecules, eventually lead to inflammation, fibrosis, and vascular dysfunction (Wei et al., 2009; Weidinger and Kozlov 2015). Of the seven NOXs isoform, NOX4 is abundantly expressed in mesangial and tubular cells and is considered the major source of ROS production in renal cells in diabetes (Sedeek et al., 2013).

Previous studies had shown that Ang II, ROS, and hyperglycemia could activate the extracellular regulated kinase (ERK) pathway in different types of renal cells, including podocytes and mesangial cells (MCs) (Gorin et al., 2004; 
Lakshmanan et al., 2012; Teng et al., 2014). ERK plays a key role in the intracellular signaling pathway that integrates the transcription of genes involved in numerous cellular responses in DN (Ni et al., 2015; Cheng et al., 2013). It act as an upstream regulator of TGF-B and SMAD pathways which are involved in renal fibrosis, ECM deposition, inflammation, and proliferation, resulting in hypertrophic response in kidney tissue (Ni et al., 2015; Lakshmanan et al., 2012; Cheng et al., 2013; He et al., 2016).

Recent studies pointed out to the role of modulating RAS activity through blocking the generation and action of Ang II using angiotensin-converting enzyme inhibitors (ACEIs) or Ang II receptor blockers (ARBs), in the management of diabetic nephropathy (Seferovic et al., 2017).

Valsartan, a non-peptide triazole derivative antagonist of Ang II, selectively and competitively blocks the binding of angiotensin II to the AT1 subtype receptor in vascular smooth muscle and the adrenal gland (S-i et al., 2011). In type 2 diabetes rat model, administration of high doses of valsartan reduces podocyte injury and renal oxidative stress, which results in not only reduced albuminuria but also arrest the progression of associated glomerulosclerosis (Zhou et al., 2014; Wang et al., 2014).

The role of valsartan in the prognosis of type 1 associated diabetic nephropathy has been less studied. Therefore, the present study was conducted to investigate the effect of valsartan on NOX4-mediated ROS production and ERK signaling pathway in type 1 diabetes rat model.

\section{Methods}

\section{Experimental animals and treatment}

Sixty adult male white albino rats (weight 200-300 g), obtained from the National research center animal house (Cairo, Egypt), were included in this study. The rats were housed in pathogen-free conditions (five rats/ cage), with 12:12 h's light-dark cycles with a stable ambient temperature of $18-22{ }^{\circ} \mathrm{C}$ in the animal house of Medicine faculty (Suez Canal University). Animals were provided with normal standard diet of known composition and free access to water. Animals were left to acclimatize for 7 days before the experiment. Animal care before and during the experimental procedures was done in accordance with the guidelines of the Animal Ethics Committee, Faculty of Medicine, Suez Canal University (license number: 2586).

Rats were divided into three groups (20 rats/group): group I (GpI) control healthy untreated rats, group II (GpII) streptozotocin (STZ)-induced diabetic rats as diseased control, and group III (GPIII) valsartan-treated STZ-induced diabetic rats.

Forty rats (comprising GpII and GpIII) were fasted overnight and rendered diabetic by a single intra-peritoneal injection of STZ purchased from Sigma-Aldrich
Chemical Company (St. Louis, MO, USA) in a dose of $65 \mathrm{mg} / \mathrm{kg} /$ body weight (BW) (Zhang et al., 2015). Rats had received $5 \%$ glucose instead of water for the first $24 \mathrm{~h}$ after diabetes induction in order to reduce death due to hypoglycemic shock, resulting from sudden hyperinsulinemia caused by massive beta cell destruction (Saeed et al., 2012). Tail vein blood glucose level were measured 3 days after STZ injection, rats with a fasting blood glucose level $\geq 250 \mathrm{mg} / \mathrm{dl}$ for three consecutive determinations were considered as diabetics (Zhang et al., 2015; Hartner et al., 2014). Two weeks later, 20 animals were presented with microalbuminuria, an early sign of DN; GpIII received intragastric valsartan (purchased from the market) at a daily dose of $30 \mathrm{mg} / \mathrm{kg} / \mathrm{BW}$ for 4 weeks.

\section{Samples collection and preparation}

At the end of the experiment, all rats were kept in metabolic cage for 24-h urine collection that is stored at $80{ }^{\circ} \mathrm{C}$ after centrifugation. Rats were fasted overnight and sacrificed under anesthesia. Blood samples were collected, kept on ice for $1 \mathrm{~h}$, and centrifuged to separate serum. Serum samples were stored at $-80{ }^{\circ} \mathrm{C}$ until analysis.

Rats' kidney tissues were dissected, rinsed in ice-cold PBS, and weighted. Left kidneys were fixed in $4 \%$ paraformaldehyde for histological studies. The right kidneys were excised into two parts: one part $(30 \mathrm{mg})$ was frozen instantly in $-80^{\circ} \mathrm{C}$ for RNA extraction, the second part was rinsed in ice-cold phosphate-buffered saline (PBS) to remove excess blood, minced to small pieces, and homogenized in $5-10 \mathrm{~mL}$ of cold PBS according to weight with a homogenizer (SR30 model ps 80) on ice. The resulting suspension was subjected to two freeze-thaw cycles, and the homogenates were centrifuged in a cooling centrifuge for $5 \mathrm{~min}$ at $5000 \times \mathrm{g}$. Supernatant was removed, aliquoted, and stored at $-80{ }^{\circ} \mathrm{C}$ for biochemical and molecular analysis.

\section{Determination of serum glucose level and kidney function markers}

Glucose, creatinine, and urea in serum and urinary creatinine concentration were assayed using automated analyzer (Hitachi, Japan, model7020) with specific commercial kits (Cobas 6000, cobas c501, Roche). Urinary microalbuminuria (UAlb) was assayed using immuneturibidemtric assay, urine albumin/urine creatinine (Ua/ Ucr) ratio, and creatinine clearance was calculated.

\section{Renal histopathological examination}

Sections (4- $\mu$ m-thick) cut from $10 \%$ formalin-fixed, paraffin-embedded kidney samples were stained with Hematoxylin and Eosin (H\&E) and Masson Trichrome staining, for evaluation of renal glomerular damage, 
tubulo-interstitial damage (Cao et al., 2000) and degree of fibrosis (Sarhan et al., 2014).

\section{RNA extraction and quantitative RT-PCR detection of renal NOX4 and ERK1/2 genes}

Thirty milligrams of renal tissues were used for total RNA extraction using RNeasy Mini Kit (cat no 74104, Qiagen, Germany). RNA was quantified using Nanodrop 8000 (Thermo Scientific USA) and cDNA was done using 10 ng RNA by reverse transcription using (QuantiTect Reverse Transcription Kit cat no205311, Qiagen) in a mastercycler gradient thermocycler [Eppendorf, Hamburg, Germany]. Then, $1 \mu \mathrm{l}$ of cDNA $(50 \mathrm{ng} / \mu \mathrm{l})$ was subjected to PCR amplification performed with an ABI PRISM 7000 Sequence Detector System (Applied Bio system). The assay reactions were performed in $25 \mu \mathrm{l}$ reaction volume including $12.5 \mu \mathrm{l}$ SYBR Green PCR mastermix (QuantiTect SYBR Green PCR Kit cat no.204141, Qiagen, Germany) according to the manufacturers' instructions, using specific sequence primers: Primers for ERK1/2 (QuantiTect primer assay, Qiagen, Cat no: QT00176330), NOX4 (QuantiTect primer assay, Qiagen, Cat no: QT00186550), and B-actin (QuantiTect primer assay, Qiagen, Cat no: QT00193473) under the following conditions: initial denaturation at $95{ }^{\circ} \mathrm{C}$ for $15 \mathrm{~min}$, followed by 45 cycles of denaturation at $95{ }^{\circ} \mathrm{C}$ for $15 \mathrm{~s}$, annealing at $56{ }^{\circ} \mathrm{C}$ for $30 \mathrm{~s}$, and extension at $72{ }^{\circ} \mathrm{C}$ for $40 \mathrm{~s}$. The results were analyzed using the $2^{-\Delta \Delta C t}$ method of analysis as described by Livak and Schmittgen (2001).

Determination of pERK and NOX4 proteins concentration and malondialdehyde level in kidney tissue homogenate Quantitative measurement of NOX4 and phosphorylated ERK1/2 (pERK1/2) in kidney tissue homogenate were carried out using NOX4 ELISA kits (cat.no. SEB924Ra cloude-clone corp. USA) and pERK ELISA kits (Instant one $^{\mathrm{Tw}}$ ELISA, cat.no. 85-86012 eBioSience, Germany) respectively according to manufacturer's instructions. Level of malondialdehyde (marker for lipid per-oxidation, and reflect the level of oxidative stress), was measured using a colorimetric assay with malondialdehyde (MDA) kit (cat no MD 2529) according to manufacturer's instructions.

\section{Statistical analysis}

All statistical comparisons were made by means of the one-way ANOVA test followed by Bonferroni post-hoc test. Data were expressed as the mean \pm standard error of the mean (SEM) and were analyzed with SPSS statistical software version 20. Chi-square was used to compare histopathological scoring and to find significance between groups. A $p$ value of $<0.05$ was considered as statistically significant. Tables and figures were produced using Microsoft Excel 2013 software.

\section{Results \\ Effect of valsartan treatment on general and metabolic parameters}

The effect of valsartan on general and metabolic parameters was shown in Table 1. There was no statistically significant difference in body weight between the three groups at the start point or at the end point of the experiment, although the diabetic group GpII lose weight and treated GpIII group gain weight but the difference was not significant. Fluid and food intake were highly significantly increased in GpII $(p=0.001)$ while fluid and food intake in GpIII was highly significantly reduced $(p=0.001)$.

Rats in GpII showed increased kidney weight that was significantly higher compared to $\mathrm{GpI}(p=0.001)$, while kidney weight in GpIII was significantly lowered than GpII $(p=0.001)$. Regarding kidney weight/body weight $(\mathrm{KW} / \mathrm{BW})$ ratio, the highest ratio was in GpII, that was significantly higher than GpI $(p=0.001)$. GpIII showed statistically significant lowered $\mathrm{KW} / \mathrm{BW}$ ratio compared to $\operatorname{GpII}(p=0.001)$.

At the end point of the experiment, GpII rats showed significantly elevated blood glucose level (hyperglycemia) $(p=0.001)$ compared to GI and valsartan treated group GpIII which showed significantly reduced blood glucose level $(p=0.001)$ compared to GpII in Fig. 1b.

\section{Effect of valsartan treatment on kidney function}

Urine volume/24 $\mathrm{h}$ in GPII diabetic rats was significantly increased compared to GpI $(p=0.002)$, while valsartan-treated GPIII showed decreased urine output compared to GpII which was statistically significant $(p=0.001)$, and urine volume of GpIII was slightly elevated than GpI with statistically non-significant difference $(p=0.735)$, Table 2 .

Valsartan-treated GPIII rats showed significant improvement of kidney function parameters compared to diabetic non-treated rats GpII, significant low levels of serum creatinine and urea compared to GpII almost approach normal value with a statistically non-significant difference compared to $\mathrm{GpI}(p=1)$. Creatinine clearance was significantly elevated in GPIII compared to GPII $(p=0.001)$ and statistically non-significant compared to GI $(p=0.140)$, Fig. 2.

On the other hand, GpII diabetic rats exhibited significant elevated level of kidney function parameters compared to GpI Fig. 2. Urinary creatinine level in GpII displayed marked reduction compared to GpI $(p=0.001)$. GpII creatinine clearance was significantly decreased compared to GpI $(p=0.001)$. 
Table 1 General characteristics of the studied groups

\begin{tabular}{llll}
\hline Groups variable (mean \pm SEM) & Gpl & Gpll & Gplll \\
\hline Body weight $(\mathrm{g})$ (start point) & $276.4 \pm 10.02$ & $266.4 \pm 9$ & $261 \pm 8$ \\
Body weight $(\mathrm{g})$ (end point) & $268.35 \pm 9.88$ & $248.05 \pm 12.21$ & $266.75 \pm 10.01$ \\
Fluid intake $(\mathrm{ml} / 24 \mathrm{~h})$ & $39.66 \pm 0.98$ & $214 \pm 10.23^{* *}$ & $64.7 \pm 1.18^{\# \#}$ \\
Food intake $(\mathrm{mg} / 24 \mathrm{~h})$ & $29.4 \pm 2.4$ & $52.16 \pm 1.88^{* *}$ & $37 \pm 1.27^{\# \#}$ \\
Kidney weight(g) & $0.49 \pm 0.02$ & $1.03 \pm 0.028^{* *}$ & $0.84 \pm 0.038^{\# \#}$ \\
KW/BW $(\mathrm{g} / \mathrm{kg})$ & $1.75 \pm 0.1$ & $4.3 \pm 0.23^{* *}$ & $3.1 \pm 0.17^{\# \#}$ \\
\hline
\end{tabular}

Values are mean \pm SEM, statistically significant difference, ${ }^{* *} p, \# \# p<0.01,(*)$ compared to Gpl: normal group, (\#) compared to Gpll: DN group. Start point: at the beginning of the experiment before induction of diabetes. End point: at the end of experiment before scarification of rat. $K W$ kidney weight, $B W$ body weight

\section{Effect of valsartan treatment on histopathological changes in kidney tissues}

Histological studies with H\&E stains showed normal renal tissues in GpI, with tubular damage score 0 , and glomerular damage score 0 (Fig. 3a).

GpII showed glomerular hypertrophy, increased mesangial cells, focal segmental sclerosis in glomerulus, congested blood vessels, and vacuolar degeneration in renal tubules. There were also interstitial inflammatory infiltrate, tubular damage: score 3+, glomerular damage: score 4+; Fig. 3b-d. GpIII showed nearly normal renal tubules, no tubular degeneration, normal Bowman's capsule, and 25\%mesangial expansion. There is no interstitial fibrosis, with residual dilatation of few tubules. Tubular damage: score $1+$, glomerular damage: score 1+; Fig. 3e, f.

Histological studies (Masson trichrome stained) for assessment of fibrosis showed no evidence of fibrosis, with a score for fibrosis: + 1 in GpI; Fig. 4a. GpII showed pronounced glomerular sclerosis and interstitial fibrosis with a score for fibrosis: + 4; Fig. 4b, c. GpIII showed minimal glomerular sclerosis and no interstitial fibrosis, with a score for fibrosis: +2 ; Fig. $4 \mathrm{~d}$.

\section{Effect of valsartan treatment on expression level of renal ERK1/2 and NOX4 genes}

The expression of NOX4 and ERK1/2 genes were both statistically significantly decreased in valsartan-treated GpIII compared to GpII $(p=0.02, p=0.001)$ Fig. 5a, b, while their expression were statistically significantly elevated in GpII compared to GpI ( $p=0.032, p=0.003)$.

\section{Effect of valsartan treatment on protein concentration of NOX4 and pERK1/2}

The highest concentration of NOX4 and pERK1/2 proteins were observed in kidneys of GPII which is statistically significant compared to $\mathrm{GpI}(p=0.001)$.

On the other hand, GpIII showed statistically significant decreased NOX4 and pERK1/2 proteins concentration compared to GpII $(p=0.001, p=0.001)$ and non-statistically significant compared to GpI $(p=1)$ Fig. $6 a$, b.

\section{Effect of valsartan on lipid peroxidation}

Malondialdehyde was significantly elevated in GpII compared to GpI $(p=0.001)$, while GpIII showed a significant decrease in MDA concentration compared to

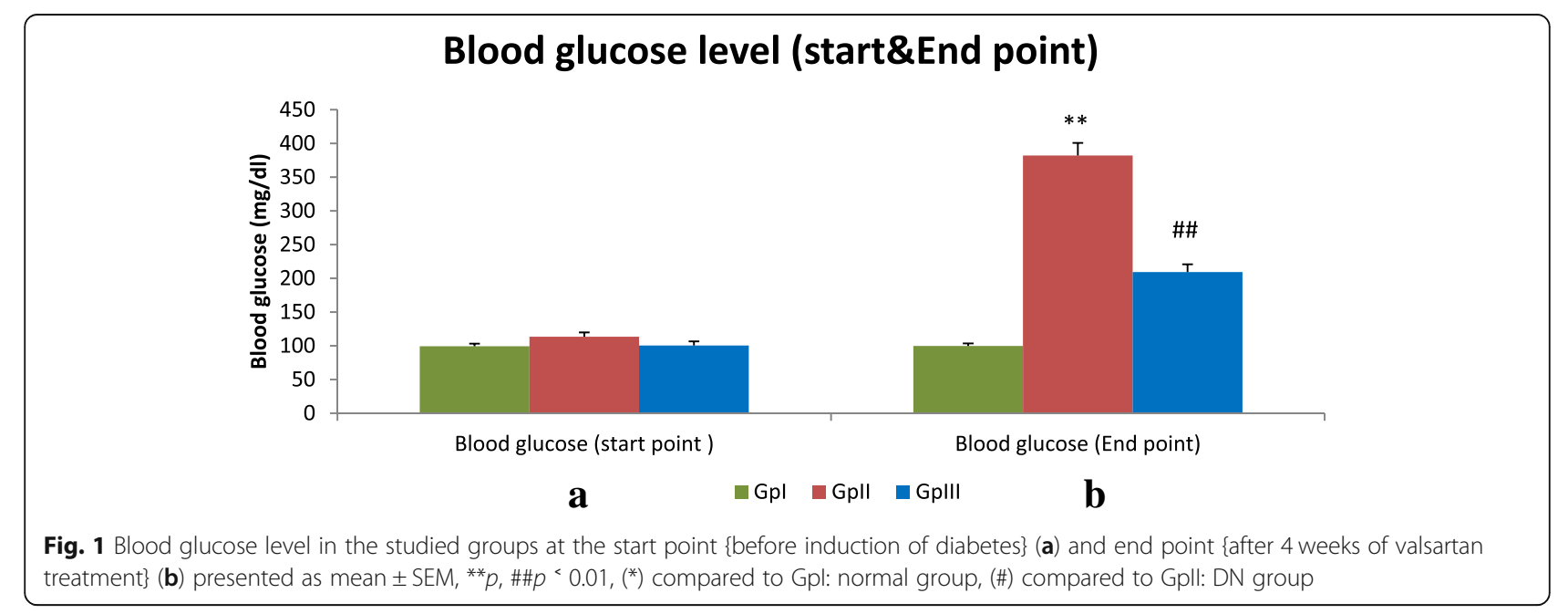


Table 2 Urine volume/ $24 \mathrm{~h}$ in the studied groups at the endpoint of treatment expressed as (mean \pm SEM)

\begin{tabular}{llll}
\hline $\begin{array}{l}\text { Groups } \\
\text { Variable }\end{array}$ & Gl & Gll & GIII \\
\hline Urine volume $(\mathrm{ml} / 24 \mathrm{~h})$ & $6.36 \pm 0.67$ & $13.93 \pm 0.27^{* *}$ & $7.10 \pm 0.30^{\# \#}$ \\
\hline
\end{tabular}

Values are mean \pm SEM, Statistically significant difference, ${ }^{* *} p$, \#\#p < $0.01,\left(^{*}\right)$ compared to Gpl: normal group, (\#) compared to Gpll: DN group
GpII ( $p=0.001)$, and statistically non-significant difference from $\mathrm{GpI}(p=0.43)$ Fig. 7.

\section{Discussion}

Diabetic nephropathy is one of the most common diabetes mellitus complication, with a heterogeneous and complex pathogenesis that involves the interaction between several factors that eventually leads to renal injury. In the present study, we investigated the effect of valsartan on the prognosis of diabetic nephropathy in type 1 diabetes rat model, induced by single STZ
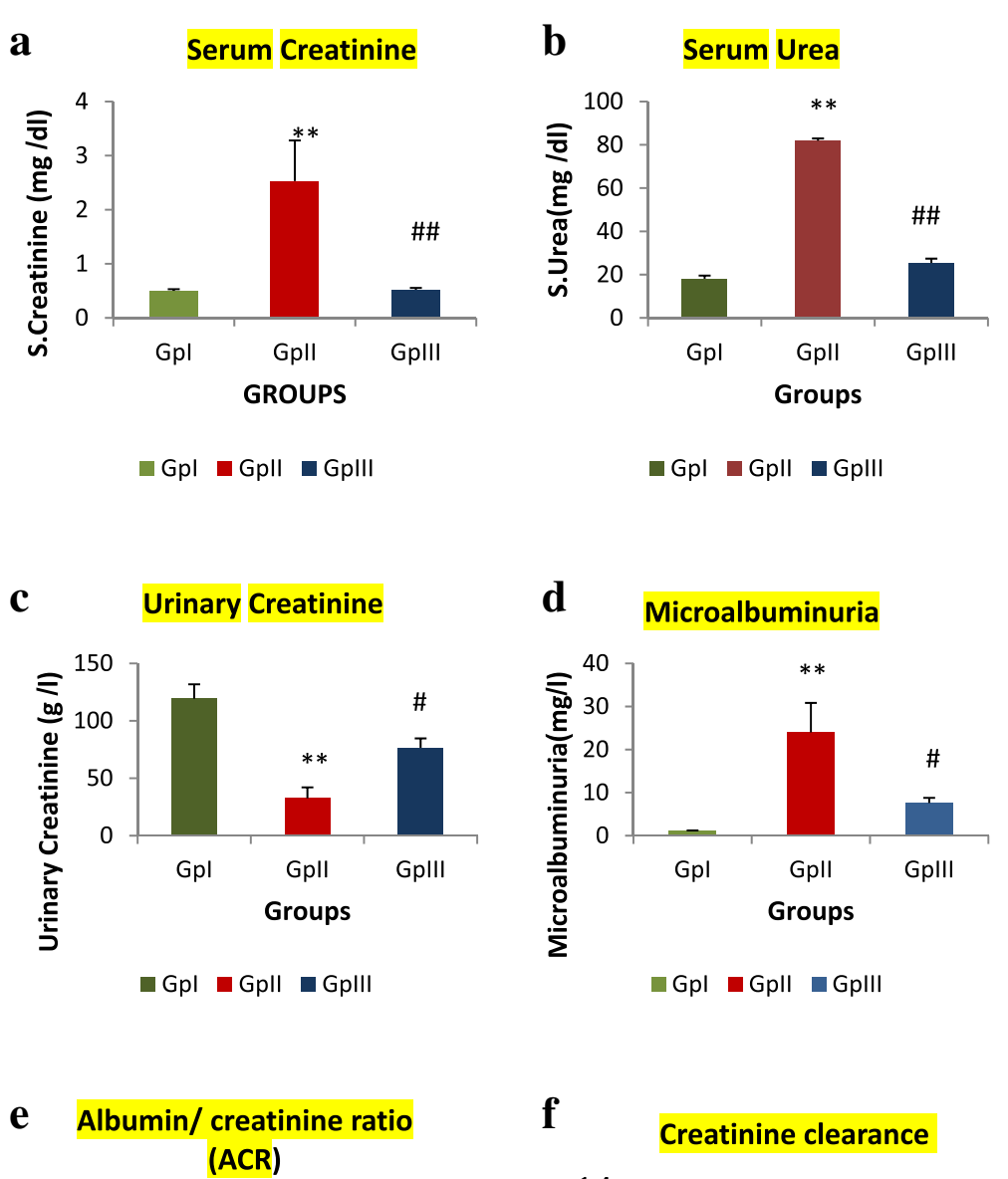

f
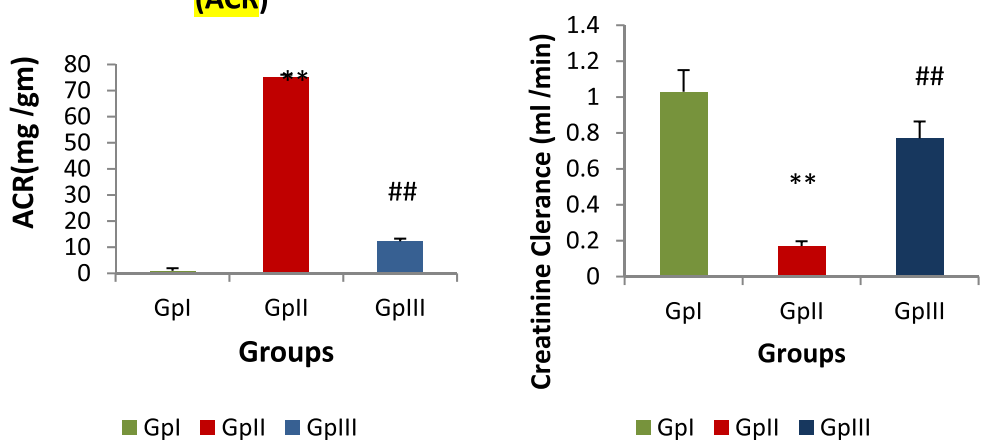

Fig. 2 Mean value of serum creatinine (a), serum urea (b), urinary creatinine (c), microalbuminuria (d), albumin creatinine ratio (e), and creatinine clearance (f), in all studied groups. ${ }^{*} p, \# p<0.05,{ }^{* *} p$, \#\#p<0.01, (*) compared to Gpl: normal group, (\#) compared to Gpll: DN group 


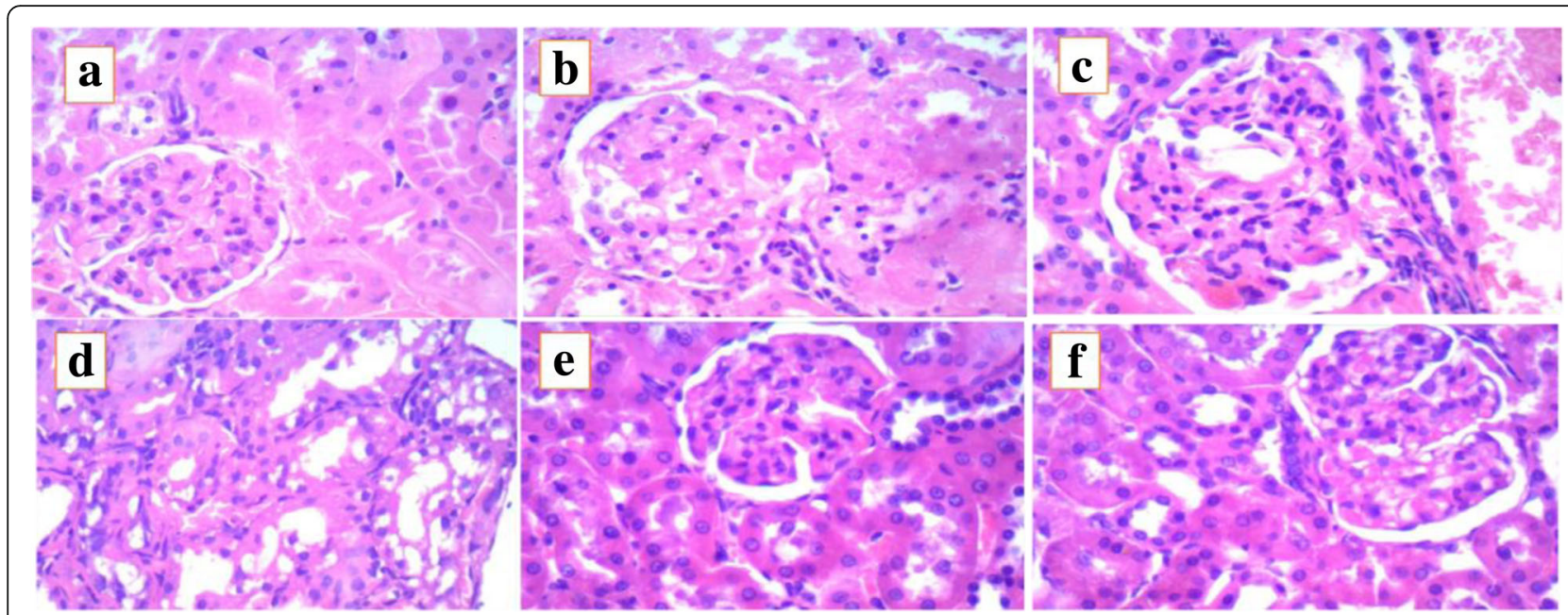

Fig. 3 Renal tissue stained with H\&E (5 mm). a Gpl: normal group. b-d Gpll: diabetic nephropathy group. e, f Gplll: valsartan-treated group

injection (Wu and Yan 2015). The kidneys of diabetic non-treated rats developed advanced pathological changes that were implicated on the kidney function parameters. This diabetic group was characterized by a highly significant increase in proteinuria which considered as a major parameter for monitoring progression of $\mathrm{DN}$, and attributed mainly to glomerular filtration barrier damage (Wang et al., 2014, 2010, 2011).

On the other hand, after 4 weeks of valsartan administration, it was evident that it restored the kidney function parameters to nearby normal levels compared to untreated group. In addition, these findings were confirmed by the histopathological study; it was noted that some histopathological changes were comparable to mild DN (class IIA) (Tervaert et al., 2010), manifested by minimal glomerular fibrosis, absence of interstitial fibrosis, nominal sclerosis, and minimal inflammation in treated diabetic rats renal tissues compared to advanced DN (class IV) in non-treated rats. Several studies reported such a beneficial effect of valsartan and other ARBs with different underlying molecular mechanisms. Wang et al., (2014) found that valsartan treatment was

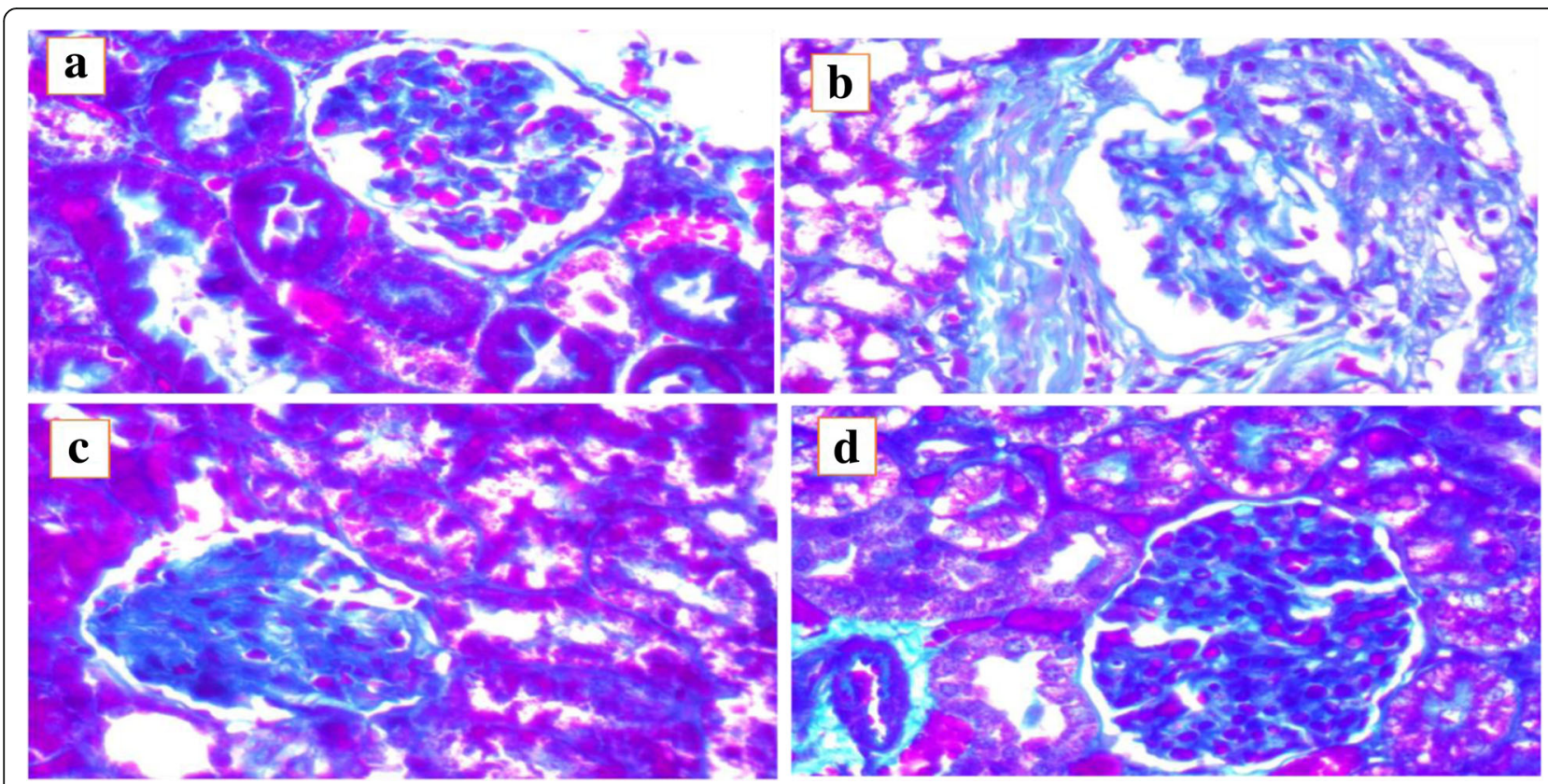

Fig. 4 Renal tissue stained with Masson trichrome $(5 \mathrm{~mm})$ from a Gpl: normal group, b, c Gpll: diabetic nephropathy group, and d Gplll: valsartan-treated group 


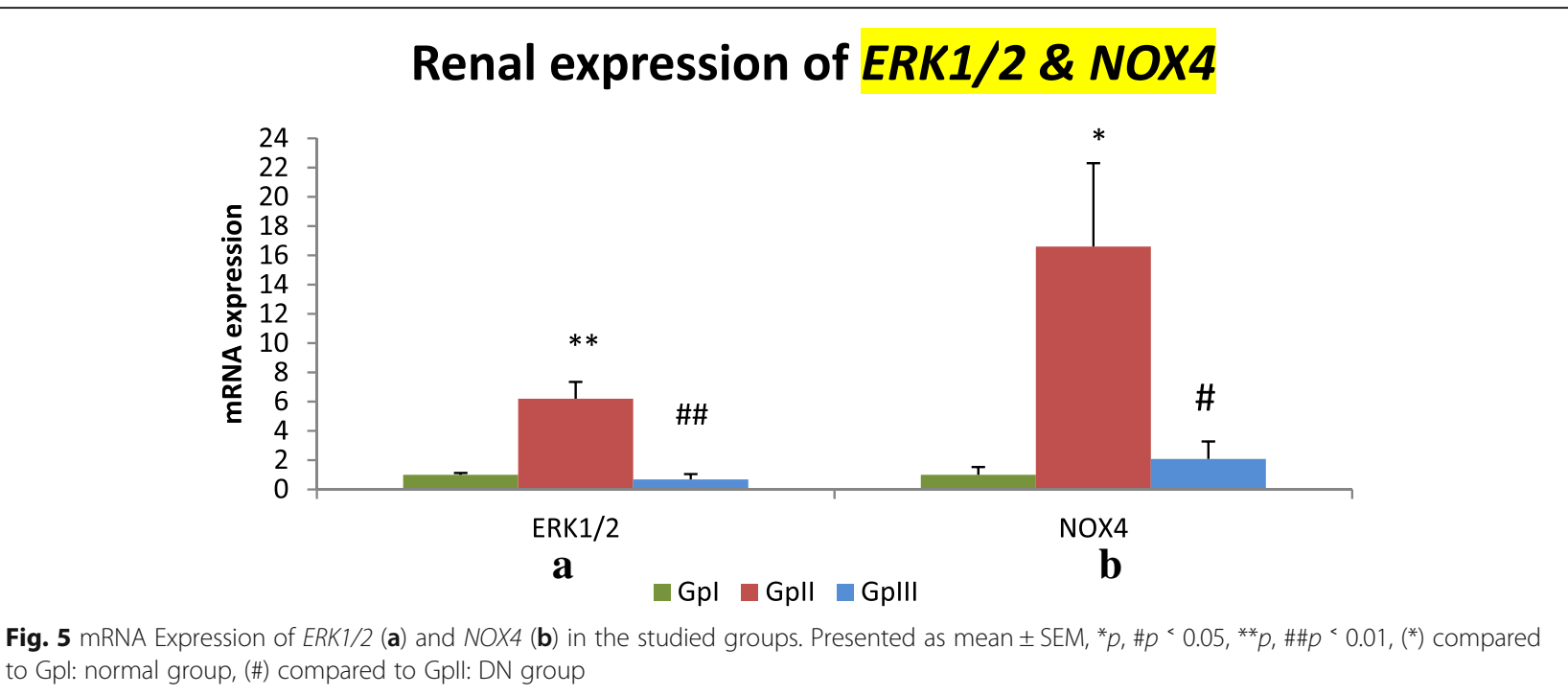

\section{a}

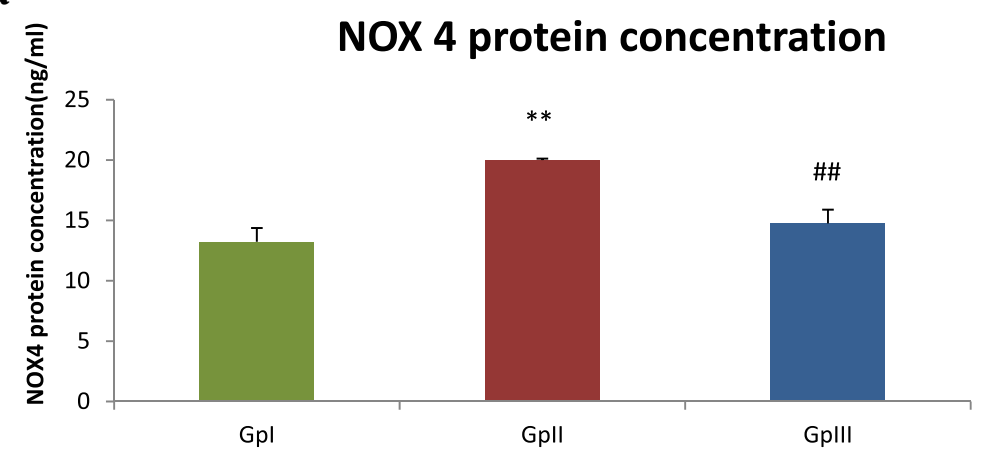

$\square \mathrm{Gpl} \square \mathrm{Gpll} \square \mathrm{GplII}$

b

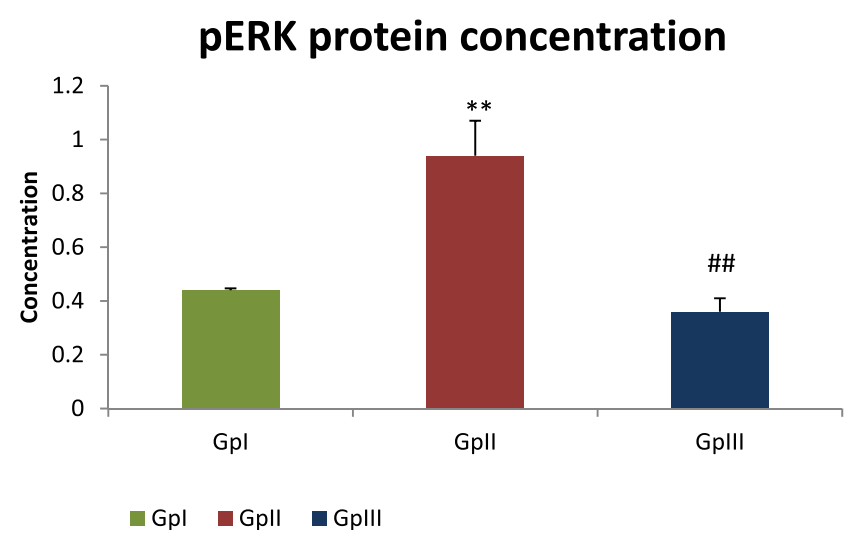

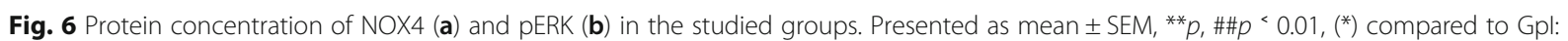
normal group, (\#) compared to Gpll: DN group 


\section{Tissue Malondialdehyde}

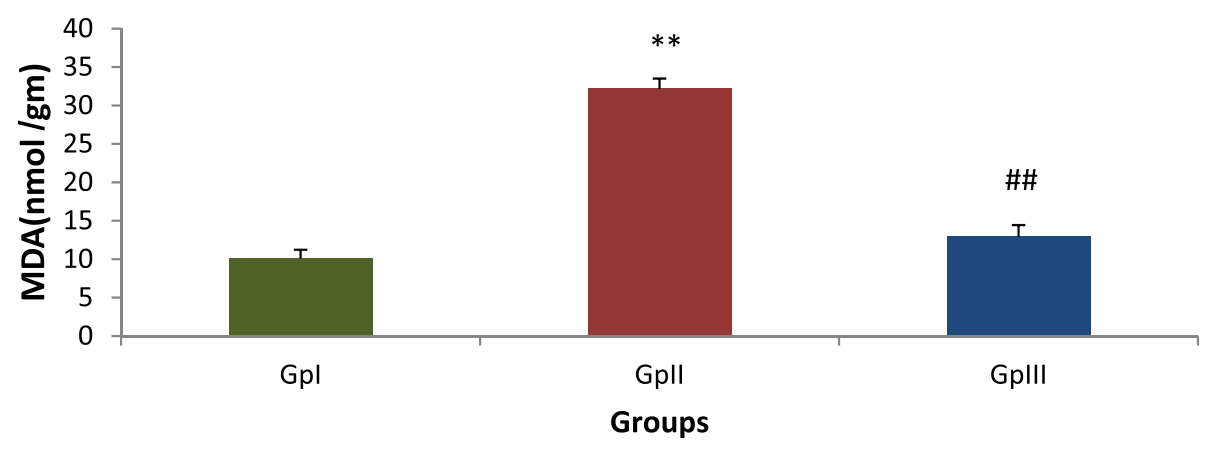

-Gpl $\quad$ Gpll $\square$ Gplll

Fig. 7 Malondialdehyde level in kidney tissue homogenate in different groups, ${ }^{* *} p, \# \# p<0.01,{ }^{* * *} p$ or \#\#\#p<0.001,(*) compared to Gpl: normal group, (\#) compared to Gpll: DN group

beneficial in protecting kidney injury in diabetes, including improvement of structural and functional changes and inhibition of fibrosis, inflammation, lipid accumulation, and ER stress in the kidney. Gao et al., (2016) reported structural and functional improvement associated with inhibition of Notch pathway and Zhou et al., (2014) related improvement to reduction in podocyte injury and renal NOX-mediated oxidative stress and inflammation. While Chen et al., (2016) relate beneficial effects of irbesartan in DN to its ability to suppress RANKL/RANK and the downstream $\mathrm{NF}_{-\mathrm{k}} \mathrm{B}$ pathway and for olmesartan, Lakshmanan et al., (2012) reveal advantageous effect by modulating MAPK cascade and NOX2 with attenuation of oxidative stress (Lakshmanan et al., 2012; Zhou et al., 2014; Wang et al., 2014; Hartner et al., 2014; Chen et al., 2016; Gao et al., 2016).

In the present study, the molecular mechanisms mediated such effects could be due to the modulation of the activity of NOX4 and pERK in the renal tissues of treated rats; diabetic non-treated rats had significantly elevated NOX4 activity evidenced by a significant increase in the gene expression and the protein concentration, accompanied by high ROS production and high MDA level in renal tissues compared to control group. Surprisingly, valsartan administration maintains NOX4 activity and MDA level within their normal values.

Previous studies confirmed that NOX4 gene expression has been increased in diabetic rodent model animal, along with elevated MDA level and increased $\mathrm{H}_{2} \mathrm{O}_{2}$ production with subsequent activation of redox-sensitive signaling pathways were previously observed in other studies and such effects were antagonized by NOX4 gene knock down by siRNA and GKT136901 NOX inhibitor (Sedeek et al., 2013; Zhou et al., 2014; Ding et al., 2007; Etoh et al.,
2003; Yaribeygi et al., 2018). In addition, $\mathrm{He}$ et al., (2016) found that ROS generation induced by high glucose concentration was mainly derived from NOX4 but not NOX1 or NOX2 in NRK-49f cells and animal model which was reversed by resveratol treatment (He et al., 2016).

Jha et al., (2014) showed that genetic deletion of NOX4 prevent the development of glomerular injury via reduction of renal ROS in diabetic mice (Jha et al., 2014). In addition, in a study on podocyte-specific deletion of NOX4, it was found that NOX4 deletion attenuated albuminuria, glomerulosclerosis, mesangial expansion, and ECM protein deposition and decreased ROS production (Jha et al., 2016), illustrating the role of NOX4 in the development of renal fibrosis.

The ability of valsartan to restraint the expression of NOX4 in renal tissues of type 1 diabetes rodent model was reported by Zhang et al., (2015), in valsartan-treated group, which points to the role of Ang II receptor blockers in inhibiting NOX4 expression in diabetic models. However, Zhou et al., (2014) observed that valsartan treatment had no effect on the elevated NOX4 expression in $d b / d b$ mice and only reduce NOX2 expression and activity. The reason for this discrepancy may be related to the different animal models used (Zhou et al., 2014; Zhang et al., 2015).

In the present study, valsartan-treated rats develop significant reduction in blood glucose level compared to non-treated DN group. The hypoglycemic effect of valsartan was observed in other studies and could be attributed to increase glucose utilization in peripheral tissues, especially muscles, through upregulation of glucose transporter 4 (GLUT4) (Guan et al., 2015; Chan et al., 2003), along with reduction in both hepatic and renal gluconeogenesis via reduce activity of phosphoenolpyruvate carboxykinase (PEPCK) via the downregulating effect of 
valsartan on NOX4-derived ROS and ERK1/2 (Winiarska et al., 2011, 2015). Conversely, several studies reported that valsartan had no effect on blood glucose level in treated compared to non-treated group; one proposed explanation for the controversy regarding valsartan effect on blood glucose may be due to experimental changes to model used (Zhou et al., 2014; Wang et al., 2014; Zhang et al., 2015; Gao et al., 2016; Sun et al., 2015).

Previous data declared that hyperglycemia could increase the expression of NOX4 mRNA in both direct and indirect pathways. The direct effect is through activation of the NF-kB pathway, mediated by $\mathrm{p} 62 / \mathrm{PK} \zeta$ interaction (Xi et al., 2015). The indirect effect achieved through induction of Ang II, that affect the mitochondrial $\mathrm{K}_{\text {АTP }}$ channels thereby simulates the expression of mitochondrial Nox4, results in abrupt production of mitochondrial superoxide and $\mathrm{H}_{2} \mathrm{O}_{2}$ in renal tubular cells (Kim et al., 2012). Similarly, ERK pathway is considered as one of the redox signaling sensitive pathways that could be activated by ROS directly or via hyperglycemia (He et al., 2016; Robinson and Cobb 1997; Fakhruddin et al., 2017; Manda et al., 2015). $\mathrm{H}_{2} \mathrm{O}_{2}$ mediate ligand-independent phosphorylation and activation of epidermal growth factor receptors, leading to activation of mitogen-activated protein 3 kinase (MAP3K), which results in ERK activation (Meves et al., 2001).

Another possible mechanism for ERK activation by ROS may comprise the inactivation and degradation of the mitogen kinase phosphatase (MKPs) that keep the pathway in an inactive state (Kamata et al., 2005). In addition, Choi et al., showed that hyperglycemia, through glutamate-induced oxidative stress, induces sustained activation of ERK pathway through a mechanism that involves degradation of MKP-1 (Choi et al., 2006).

It was previously reported that ERK $1 / 2$ was the main mediator of renal fibrosis, ECM deposition, inflammation, and proliferation in high glucose environment through regulation of TGF-B and SMAD pathways (Ni et al., 2015; Cheng et al., 2013; He et al., 2016; Abe et al., 2002), and has an important role in glomerular-endothelial-mesenchymal transition that characterizes DN (He et al., 2015; Shang et al., 2017).

In the present study, the ERK1/2 mRNA expression as well as pERK1/2 protein concentration were significantly elevated in diabetic non-treated group compared to control. A result that was consistent with other previous studies (Sedeek et al., 2013; Lakshmanan et al., 2012; Cheng 2013). Moreover, following valsartan administration, ERK1/2 mRNA expression and pERK1/2 protein concentration were significantly reduced, and such an effect was most likely mediated through Ang II blockage, NOX4-derived oxidative stress reduction, and the hypoglycemic effect.

\section{Conclusions}

In conclusion, valsartan could protect the kidney from the deleterious effect of type 1 diabetes-associated nephropathy progression through decreasing oxidative stress, ERK1/2, and NOX4 activity. Early interventions by valsartan to decrease the rate of GFR loss could prolong the time to development of diabetic nephropathy and ultimately end-stage renal disease (ESRD).

\section{Abbreviations}

Ang II: Angiotensin II; DN: Diabetic nephropathy; ERK: The extracellular regulated kinase pathway; ESRD: End stage renal disease; MCs: Mesangial cells; MDA: Malondialdehyde; MKPs: Mitogen kinase phosphatase; NADPH: Nicotinamide adenine dinucleotide phosphate; NOX4: NADPH oxidase 4; PEPCK: Phosphoenolpyruvate carboxykinase; RAS: Intrarenal reninangiotensin system; ROS: Reactive oxygen species; STZ: Streptozotocin

\section{Acknowledgments}

The authors thanks Dr. Mohamed Kamal lecturer of pathology for interpreting pathological finding, Medical Biochemistry and Molecular Biology department, Physiology department, Center of Excellence for Cellular and Molecular Medicine, Faculty of Medicine, Suez Canal University.

Funding

Self-funding

\section{Availability of data and materials}

The data used to support the findings of this study are available from the corresponding author upon request.

\section{Authors' contributions}

SHAM, LATE, and DIB conceived and designed the experiments. SHAM collected samples and data. SHAM and LATE performed the experiments. DIB analyzed the data. SAM, LATE, and DIB wrote the manuscript. SHAM, LATE, DIB, and TIE were responsible for the final revision and approval of the manuscript. All authors read and approved the final manuscript.

Ethics approval and consent to participate

All the experimental protocols were approved by the institutional research ethics committee at Faculty of Medicine, Suez Canal University.

Consent for publication

Not applicable

\section{Competing interests}

The authors declare that they have no competing interests.

\section{Publisher's Note}

Springer Nature remains neutral with regard to jurisdictional claims in published maps and institutional affiliations.

Received: 7 May 2019 Accepted: 8 August 2019

Published online: 30 August 2019

\section{References}

Abe MK, Saelzler MP, Espinosa R, Kahle KT, Hershenson MB, Le Beau MM et al (2002) ERK8, a new member of the mitogen-activated protein kinase family. J Biol Chem 277(19):16733-16743

Cao Z, Cooper ME, Wu LL, Cox AJ, Jandeleit-Dahm K, Kelly DJ et al (2000) Blockade of the renin-angiotensin and endothelin systems on progressive renal injury. Hypertension. 36(4):561-568

Chan P, Wong K-L, Liu I-M, Tzeng T-F, Yang T-L, Cheng J-T (2003) Antihyperglycemic action of angiotensin II receptor antagonist, valsartan, in streptozotocin-induced diabetic rats. J Hypertens 21(4):761-769

Chen X-W, Du X-Y, Wang Y-X, Wang J-C, Liu W-T, Chen W-J et al (2016) Irbesartan ameliorates diabetic nephropathy by suppressing the RANKLRANK-NF-KB pathway in type 2 diabetic db/db mice. Mediat Inflamm 2016:10 pages 
Cheng X, Gao W, Dang Y, Liu X, Li Y, Peng X et al (2013) Both ERK/MAPK and TGFBeta/Smad signaling pathways play a role in the kidney fibrosis of diabetic mice accelerated by blood glucose fluctuation. J Diabetes Res 2013:8 pages

Choi B-H, Hur E-M, Lee J-H, Jun D-J, Kim K-T (2006) Protein kinase C C-mediated proteasomal degradation of MAP kinase phosphatase-1 contributes to glutamate-induced neuronal cell death. J Cell Sci 119(7):1329-1340

Collins AJ, Foley RN, Chavers B, Gilbertson D, Herzog C, Johansen K et al (2012) US renal data system 2011 annual data report. Am J Kidney Dis 59(1):A7

Ding H, Hashem M, Triggle C (2007) Increased oxidative stress in the streptozotocin-induced diabetic apoE-deficient mouse: changes in expression of NADPH oxidase subunits and eNOS. Eur J Pharmacol 561(1):121-128

Etoh T, Inoguchi T, Kakimoto M, Sonoda N, Kobayashi K, Kuroda J et al (2003) Increased expression of NAD (P) H oxidase subunits, NOX4 and p22phox, in the kidney of streptozotocin-induced diabetic rats and its reversibity by interventive insulin treatment. Diabetologia. 46(10):1428-1437

Fakhruddin S, Alanazi W, Jackson KE (2017) Diabetes-induced reactive oxygen species: mechanism of their generation and role in renal injury J Diabetes Res 2017:30 pages

Gao F, Yao M, Cao Y, Liu S, Liu Q, Duan H (2016) Valsartan ameliorates podocyte loss in diabetic mice through the notch pathway. Int J Mol Med 37(5):1328-1336

Gnudi L, Coward RJ, Long DA (2016) Diabetic nephropathy: perspective on novel molecular mechanisms. Trends Endocrinol Metab 27(11):820-830

Gorin Y, Ricono JM, Wagner B, Nam-Ho K, Bhandari B, Choudhury GG et al (2004) Angiotensin II-induced ERK1/ERK2 activation and protein synthesis are redoxdependent in glomerular mesangial cells. Biochem J 381(1):231-239

Guan J, Liu W-Q, Xing M-Q, Shi Y, Tan X-Y, Jiang C-Q et al (2015) Elevated expression of periostin in diabetic cardiomyopathy and the effect of valsartan. BMC Cardiovasc Disord 15(1):90

Hartner A, Cordasic N, Klanke B, Menendez-Castro C, Veelken R, Schmieder RE et al (2014) Renal protection by low dose irbesartan in diabetic nephropathy is paralleled by a reduction of inflammation, not of endoplasmic reticulum stress. Biochim Biophys Acta (BBA)-Mol Basis Dis 1842(4):558-565

He T, Guan X, Wang S, Xiao T, Yang K, Xu X et al (2015) Resveratrol prevents high glucose-induced epithelial-mesenchymal transition in renal tubular epithelial cells by inhibiting NADPH oxidase/ROS/ERK pathway. Mol Cell Endocrinol 402:13-20

He T, Xiong J, Nie L, Yu Y, Guan X, Xu X et al (2016) Resveratrol inhibits renal interstitial fibrosis in diabetic nephropathy by regulating AMPK/NOX4/ROS pathway. J Mol Med 94(12):1359-1371

Jha JC, Gray SP, Barit D, Okabe J, El-Osta A, Namikoshi T et al (2014) Genetic targeting or pharmacologic inhibition of NADPH oxidase nox4 provides renoprotection in long-term diabetic nephropathy. J Am Soc Nephrol 25(6):1237-1254

Jha JC, Thallas-Bonke V, Banal C, Gray SP, Chow BS, Ramm G et al (2016) Podocyte-specific Nox4 deletion affords renoprotection in a mouse model of diabetic nephropathy. Diabetologia. 59(2):379-389

Kamata H, Honda S-I, Maeda S, Chang L, Hirata H, Karin M (2005) Reactive oxygen species promote TNFa-induced death and sustained JNK activation by inhibiting MAP kinase phosphatases. Cell. 120(5):649-661

Kim S-M, Kim Y-G, Jeong K-H, Lee S-H, Lee T-W, Ihm C-G et al (2012) Angiotensin Il-induced mitochondrial Nox4 is a major endogenous source of oxidative stress in kidney tubular cells. PLoS One 7(7):e39739

Lakshmanan AP, Thandavarayan RA, Watanabe K, Sari FR, Meilei H, Giridharan W et al (2012) Modulation of AT-1R/MAPK cascade by an olmesartan treatment attenuates diabetic nephropathy in streptozotocin-induced diabetic mice. Mol Cell Endocrinol 348(1):104-111

Livak KJ, Schmittgen TD (2001) Analysis of relative gene expression data using real-time quantitative PCR and the $2-\Delta \Delta C T$ method. Methods 25(4):402-408

Manda G, Checherita A-I, Comanescu MV, Hinescu ME (2015) Redox signaling in diabetic nephropathy: hypertrophy versus death choices in mesangial cells and podocytes. Mediat Inflamm 2015:13

Meves A, Stock SN, Beyerle A, Pittelkow MR, Peus D (2001) H2O2 mediates oxidative stress-induced epidermal growth factor receptor phosphorylation. Toxicol Lett 122(3):205-214

Murphy AM, Wong AL, Bezuhly M (2015) Modulation of angiotensin II signaling in the prevention of fibrosis. Fibrogenesis Tissue Repair 8(1):7

Ni WJ, Tang LQ, Wei W (2015) Research progress in signalling pathway in diabetic nephropathy. Diabetes Metab Res Rev 31(3):221-233
Onozato ML, Tojo A, Goto A, Fujita T, Wilcox CS (2002) Oxidative stress and nitric oxide synthase in rat diabetic nephropathy: effects of ACEI and ARB. Kidney Int 61(1):186-194

Robinson MJ, Cobb MH (1997) Mitogen-activated protein kinase pathways. Curr Opin Cell Biol 9(2):180-186

Saeed S, Mosa-Al-Reza H, Fatemeh AN, Saeideh D (2012) Antihyperglycemic and antihyperlipidemic effects of guar gum on streptozotocin-induced diabetes in male rats. Pharmacogn Mag 8(29):65

Sarhan M, El Serougy H, Hussein AM, El-Dosoky M, Sobh MA, Fouad SA et al (2014) Impact of bone-marrow-derived mesenchymal stem cells on adriamycin-induced chronic nephropathy. Can J Physiol Pharmacol 92(9):733-743

Sedeek M, Gutsol A, Montezano AC, Burger D, Cat AND, Kennedy CR et al (2013) Renoprotective effects of a novel Nox1/4 inhibitor in a mouse model of type 2 diabetes. Clin Sci 124(3):191-202

Seferovic JP, Claggett B, Seidelmann SB, Seely EW, Packer M, Zile MR et al (2017) Effect of sacubitril/valsartan versus enalapril on glycaemic control in patients with heart failure and diabetes: a post-hoc analysis from the PARADIGM-HF trial. Lancet Diabetes Endocrinol 5(5):333-340

Shang J, Zhang Y, Jiang Y, Li Z, Duan Y, Wang L et al (2017) NOD2 promotes endothelial-to-mesenchymal transition of glomerular endothelial cells via MEK/ERK signaling pathway in diabetic nephropathy. Biochem Biophys Res Commun 484(2):435-441

S-i M, Karnik SS, Saku K (2011) Angiotensin II type 1 receptor blockers: class effects versus molecular effects. J Renin-Angiotensin-Aldosterone Syst 12(1):1-7

Singh R, Alavi N, Singh AK, Leehey DJ (1999) Role of angiotensin II in glucose-induced inhibition of mesangial matrix degradation. Diabetes. 48(10):2066-2073

Sun H, Zhao Y, Bi X, Li S, Su G, Miao Y et al (2015) Valsartan blocks thrombospondin/transforming growth factor/Smads to inhibit aortic remodeling in diabetic rats. Diagn Pathol 10(1):18

Teng B, Duong M, Tossidou I, Yu X, Schiffer M (2014) Role of protein kinase C in podocytes and development of glomerular damage in diabetic nephropathy. Front Endocrinol 5:179

Tervaert TWC, Mooyaart AL, Amann K, Cohen AH, Cook HT, Drachenberg CB et al (2010) Pathologic classification of diabetic nephropathy. J Am Soc Nephrol 21(4):556-563

Urushihara M, Kagami S (2017) Role of the intrarenal renin-angiotensin system in the progression of renal disease. Pediatr Nephrol 32(9):1471-1479

Ushio-Fukai M (2009) Vascular signaling through G protein coupled receptorsnew concepts. Curr Opin Nephrol Hypertens 18(2):153

Wang W, Qiu L, Howard A, Solis N, Li C, Wang X et al (2014) Protective effects of aliskiren and valsartan in mice with diabetic nephropathy. J ReninAngiotensin-Aldosterone Syst 15(4):384-395

Wang XX, Jiang T, Shen Y, Caldas Y, Miyazaki-Anzai S, Santamaria H et al (2010) Diabetic nephropathy is accelerated by farnesoid $X$ receptor deficiency and inhibited by farnesoid $X$ receptor activation in a type 1 diabetes model. Diabetes. 59:2916-2927

Wang $X X$, Jiang $T$, Shen $Y$, Santamaria $H$, Solis N, Arbeeny $C$ et al (2011) Vitamin $\mathrm{D}$ receptor agonist doxercalciferol modulates dietary fat-induced renal disease and renal lipid metabolism. Am J Physiol Renal Physiol 300(3):F801-FF10

Wei W, Liu Q, Tan Y, Liu L, Li X, Cai L (2009) Oxidative stress, diabetes, and diabetic complications. Hemoglobin. 33(5):370-377

Weidinger A, Kozlov AV (2015) Biological activities of reactive oxygen and nitrogen species: oxidative stress versus signal transduction. Biomolecules. 5(2):472-484

Winiarska K, Grabowski M, Rogacki MK (2011) Inhibition of renal gluconeogenesis contributes to hypoglycaemic action of NADPH oxidase inhibitor, apocynin. Chem Biol Interact 189(1-2):119-126

Winiarska K, Jarzyna R, Dzik JM, Jagielski AK, Grabowski M, Nowosielska A et al (2015) ERK1/2 pathway is involved in renal gluconeogenesis inhibition under conditions of lowered NADPH oxidase activity. Free Radic Biol Med 81:13-21

Wu J, Yan L-J (2015) Streptozotocin-induced type 1 diabetes in rodents as a model for studying mitochondrial mechanisms of diabetic $\beta$ cell glucotoxicity. Diabetes Metab Syndr Obes 8:181

Xi G, Shen X, Wai C, Vilas CK, Clemmons DR (2015) Hyperglycemia stimulates p62/PKC interaction, which mediates NF-KB activation, increased Nox4 expression, and inflammatory cytokine activation in vascular smooth muscle. FASEB J 29(12):4772-4782 
Yadav A, Vallabu S, Arora S, Tandon P, Slahan D, Teichberg S et al (2010) ANG ॥ promotes autophagy in podocytes. Am J Phys Cell Phys 299(2):C488-CC96

Yaribeygi H, Mohammadi MT, Rezaee R, Sahebkar A (2018) Fenofibrate improves renal function by amelioration of NOX-4, IL-18, and p53 expression in an experimental model of diabetic nephropathy. J Cell Biochem 119:7458-7469

Zhang K, Meng X, Li D, Yang J, Kong J, Hao P et al (2015) Angiotensin (1-7) attenuates the progression of streptozotocin-induced diabetic renal injury better than angiotensin receptor blockade. Kidney Int 87(2):359-369

Zhang S-L, Filep JG, Hohman TC, Tang S-S, Ingelfinger JR, Chan JS (1999) Molecular mechanisms of glucose action on angiotensinogen gene expression in rat proximal tubular cells. Kidney Int 55(2):454-464

Zhou G, Cheung AK, Liu X, Huang Y (2014) Valsartan slows the progression of diabetic nephropathy in $\mathrm{db} / \mathrm{db}$ mice via a reduction in podocyte injury, and renal oxidative stress and inflammation. Clin Sci 126(10):707-720

\section{Submit your manuscript to a SpringerOpen ${ }^{\circ}$ journal and benefit from:}

- Convenient online submission

- Rigorous peer review

- Open access: articles freely available online

High visibility within the field

Retaining the copyright to your article

Submit your next manuscript at $\boldsymbol{\wedge}$ springeropen.com 